\title{
Fusion of airborne LiDAR with multispectral SPOT 5 image for enhancement of feature extraction using Dempster-Shafer theory
}

\begin{abstract}
This paper presents an application of data-driven Dempster-Shafer theory (DST) of evidence to fuse multisensor data for land-cover feature extraction. Over the years, researchers have focused on DST for a variety of applications. However, less attention has been given to generate and interpret probability, certainty, and conflict maps. Moreover, quantitative assessment of DST performance is often overlooked. In this paper, for implementation of DST, two main types of data were used: multisensor data such as Light Detection and Ranging (LiDAR) and multispectral satellite imagery [Satellite Pour l'Observation de la Terre 5 (SPOT 5)]. The objectives are to classify land-cover types from fused multisensor data using DST, to quantitatively assess the accuracy of the classification, and to examine the potential of slope data derived from LiDAR for feature detection. First, we derived the normalized difference vegetation index (NDVI) from SPOT 5 image and the normalized digital surface model (DSM) (nDSM) from LiDAR by subtracting the digital terrain model from the DSM. The two products were fused using the DST algorithm, and the accuracy of the classification was assessed. Second, we generated a surface slope from LiDAR and fused it with NDVI. Subsequently, the classification accuracy was assessed using an IKONOS image of the study area as ground truth data. From the two processing stages, the $\mathrm{NDVI} / \mathrm{nDSM}$ fusion had an overall accuracy of $88.7 \%$, while the NDVI/slope fusion had 75.3\%. The result indicates that NDVI/nDSM integration performed better than NDVI/slope. Although the overall accuracy of the former is better than the latter (NDVI/slope), the contribution of individual class reveals that building extraction from fused slope and NDVI performed poorly. This study proves that DST is a time- and cost-effective method for accurate land-cover feature identification and extraction without the need for a prior knowledge of the scene. Furthermore, the ability to generate ot- er products like certainty, conflict, and maximum probability maps for better visual understanding of the decision process makes it more reliable for applications such as urban planning, forest management, 3$\mathrm{D}$ feature extraction, and map updating.
\end{abstract}

Keyword: Dempster-Shafer theory (DST); Fusion; GIS; Light Detection and Ranging (LiDAR); Remote sensing 